\title{
Boundaries determine the formation energies of lattice defects in two-dimensional buckled materials
}

\author{
Sandeep K. Jain, ${ }^{1, *}$ Vladimir Juričić, ${ }^{1,2, \dagger}$ and Gerard T. Barkema ${ }^{1,3}$ \\ ${ }^{1}$ Institute for Theoretical Physics, Universiteit Utrecht, Leuvenlaan 4, 3584 CE Utrecht, The Netherlands \\ ${ }^{2}$ Nordita, Center for Quantum Materials, KTH Royal Institute of Technology, and Stockholm University, \\ Roslagstullsbacken 23, S-106 91 Stockholm, Sweden \\ ${ }^{3}$ Instituut-Lorentz, Universiteit Leiden, Niels Bohrweg 2, 2333 CA Leiden, The Netherlands \\ (Received 4 March 2016; revised manuscript received 25 June 2016; published 19 July 2016)
}

\begin{abstract}
Lattice defects are inevitably present in two-dimensional materials, with direct implications on their physical and chemical properties. We show that the formation energy of a lattice defect in buckled two-dimensional crystals is not uniquely defined as it takes different values for different boundary conditions even in the thermodynamic limit, as opposed to their perfectly planar counterparts. Also, the approach to the thermodynamic limit follows a different scaling: inversely proportional to the logarithm of the system size for buckled materials, rather than the usual power-law approach. In graphene samples of $\sim 1000$ atoms, different boundary conditions can cause differences exceeding $10 \mathrm{eV}$. Besides presenting numerical evidence in simulations, we show that the universal features in this behavior can be understood with simple bead-spring models. Fundamentally, our findings imply that it is necessary to specify the boundary conditions for the energy of the lattice defects in the buckled two-dimensional crystals to be uniquely defined, and this may explain the lack of agreement in the reported values of formation energies in graphene. We argue that boundary conditions may also have an impact on other physical observables such as the melting temperature.
\end{abstract}

DOI: 10.1103/PhysRevB.94.020102

Lattice irregularities in the form of defects, such as dislocations and grain boundaries, are quite generically present in crystalline lattices. Usually, defects have a direct impact on the various properties of the material; for instance, in graphene they reduce the mobility [1], and change Young's modulus [2,3] and the fracture behavior [4]. A fundamental property characterizing a lattice defect is its formation energy, with the crucial importance for their behavior, e.g., the defects' migration and healing [5]. On the other hand, two-dimensional crystals have a natural tendency to buckle out of the crystalline plane to relieve the stress [6-8]. For perfectly confined twodimensional materials, the formation energy of a lattice defect does not depend on the boundary conditions, but only on the type of the defect, and in that sense is uniquely defined. However, the question arises whether this fundamentally important feature of the lattice defects changes in buckled crystals, and in particular whether the boundaries affect the defects' energy.

In this Rapid Communication, we show that this is indeed the case by studying the formation energy of the defects in both simple, analytically tractable buckled one- and twodimensional bead-spring models, as well as in numerical simulations of graphene, a paradigmatic representative of a two-dimensional buckled crystal. In particular, we find that unlike two-, and three-dimensional materials where the formation energy of a lattice defect, such as an SW defect, is well defined, in buckled two-dimensional materials different boundary conditions give rise to different values of the formation energy of the defect in the thermodynamic limit. Moreover, while the finite-size correction in the energy scales as inversely proportional to the system size for one-, two-, and three-dimensional materials, we show that this scaling

\footnotetext{
*S.K.Jain@uu.nl

†juricic@nordita.org
}

for buckled sheet-type materials is given by the inverse of logarithm of the system size.

To describe this boundary effect on the formation energy of the defects in buckled crystals, we first consider a simple model of a string of $N$ atoms with length $L=N$, connected with elastic springs and a defect created at the center of the string by making the bond angle with the $y$ axis equal to $\theta \neq 0$ [Fig. 1(a)]. This string is embedded in two-dimensional space and in this way we allow for the buckling in the model. In this $(1+1)$-dimensional $[(1+1) \mathrm{D}]$ model, the energy of the defect configuration is minimized for the two most commonly used boundary conditions: force-free (FF) boundaries which relax the global planar stress, and deformation-free (DF) boundaries which fix the density of the atoms to the crystalline density [Fig. 1(a)]. We use the Hamiltonian

$$
\begin{aligned}
H= & E_{\mathrm{core}}+\lambda \sum_{i}\left(r_{i}-1\right)^{2}+\kappa \sum_{i}\left(\phi_{i+1}-\phi_{i}\right)^{2} \\
& -f\left(\sum_{i} r_{i} \cos \left(\phi_{i}\right)-L\right)
\end{aligned}
$$

where $r_{i}$ is the bond length between two neighboring atoms $i-1$ and $i$, and $\phi_{i}$ is the angle of this bond with respect to the $x$ axis. For simplicity, we set the core energy of defect $E_{\text {core }}=0$. The elastic constants in the Hamiltonian are defined as follows: $\lambda$ is the bond stretching constant, $\kappa$ is the bond bending constant, and $f$ is the force acting on the boundaries. At the FF boundary condition the energy is minimized for $r_{i}=1$ and $\phi_{i}=\phi_{0}\left(-1+\frac{i}{N}\right)$, which leads to the finite-size energy scaling of $\sim 1 / N$. In Fig. 1(b) numerical values of FF energy calculations are shown (points) and are in very good agreement with the analytical solution (fitted with line). Furthermore, DF boundary conditions yield a minimum energy for $r_{i}=$ $1+f /(2 \lambda)$ and $\phi_{i}=\phi_{0} \exp (-\alpha i)$ with $\alpha=\sqrt{f r_{i} / 2 \kappa}$. These solutions in turn yield forces with finite-size scaling of the 
(a)

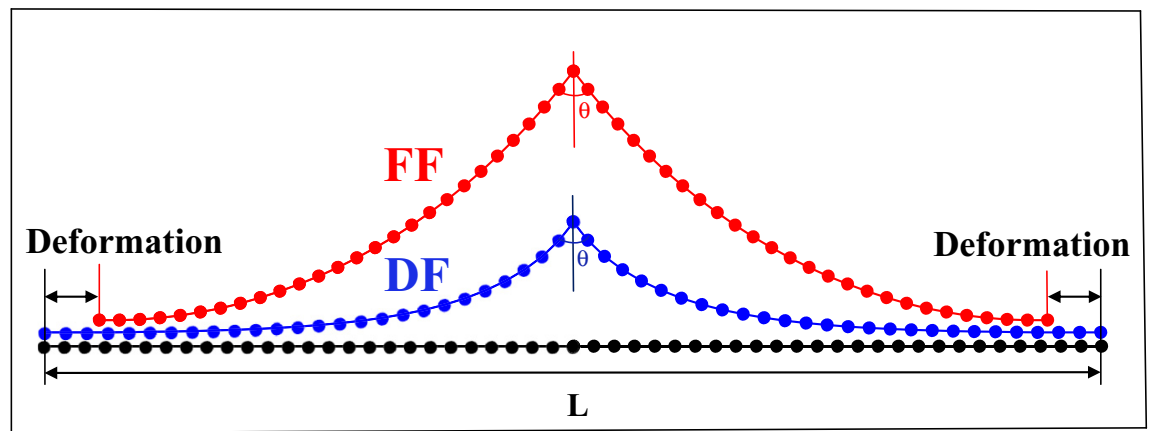

(b)

0.025

4

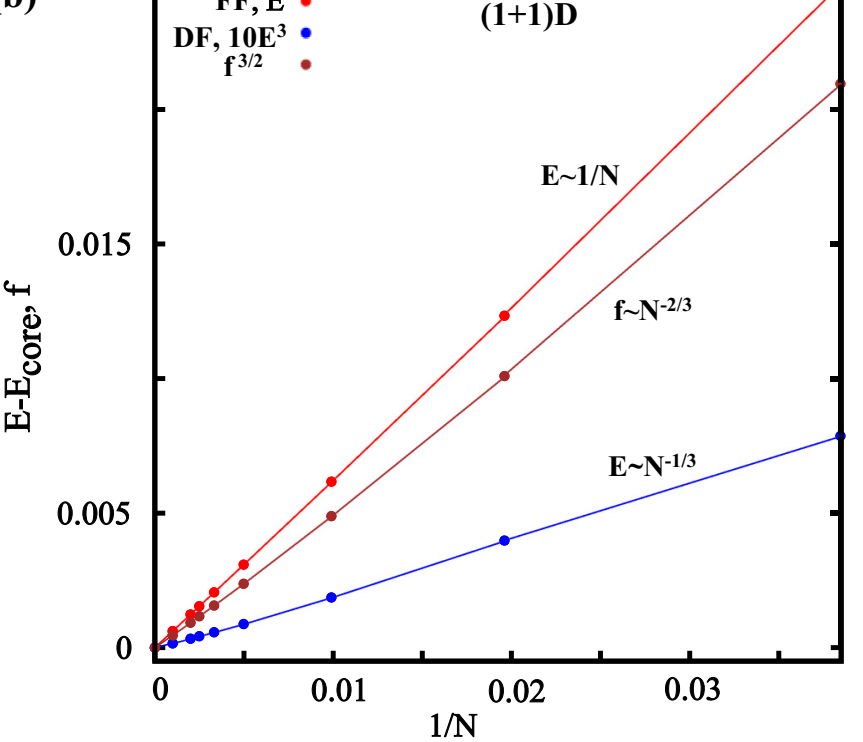

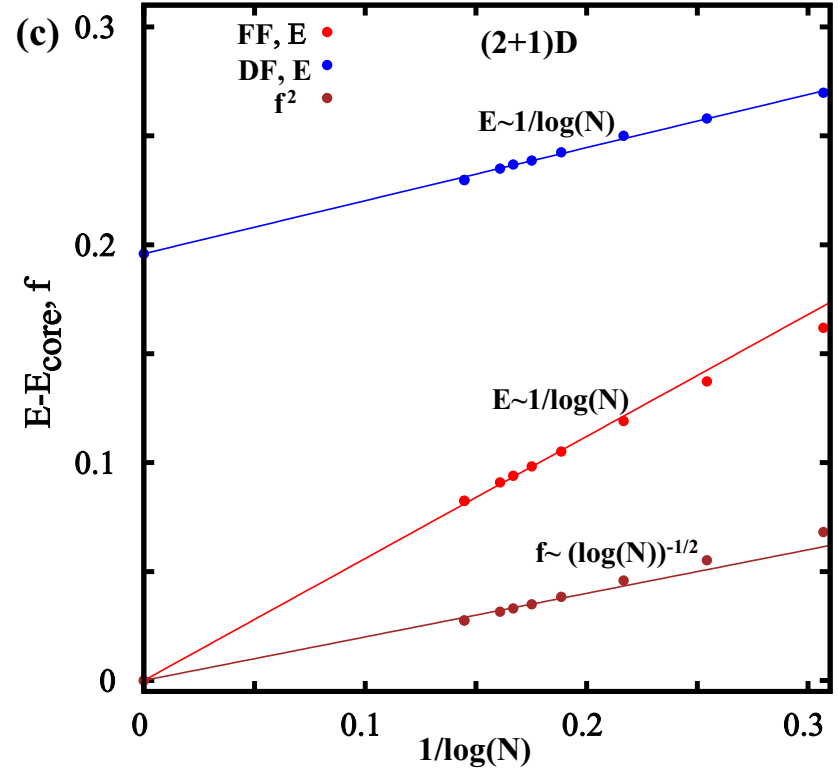

FIG. 1. Illustration of force-free (FF) and deformation-free (DF) boundaries and calculated defect energy as a function of the system size for both $(1+1) \mathrm{D}$ and $(2+1) \mathrm{D}$ models. (a) Sketch of the elastic string model that accounts for the boundary effects on the formation energy of the defects. In the case of DF boundaries, the introduction of the defect does not change the total length $(L)$ since a force is acting on the boundaries to keep the sample at constant density. In the case of FF boundaries, the density of the sample does change through the change of the length. (b) Finite-size correction to the energy and force for both the FF and DF boundaries in the $(1+1) \mathrm{D}$ model. The numerical data points are fitted well by the analytically predicted scaling of the energy for DF (blue line) and FF (red line) boundaries and force (brown line). (c) Finite-size correction to the energy and force for both the FF and DF boundaries in the (2+1)D model. The numerical data points are fitted well by the analytically predicted scaling of the energy for DF (blue line) and FF (red line) boundaries and square of the force (brown line). Here, we use the values of the parameters in the Hamiltonian $(1), \lambda=\kappa=1$.

form $f \sim N^{-2 / 3}$, while the energy scales as $E \sim N^{-1 / 3}$. We have also performed the numerical simulations for DF boundary conditions, and these results are in agreement with the analytical ones [Fig. 1(b)]. More importantly, this very simple model already yields a different scaling of the energy with the system size for different boundaries, a feature also prominent in the $(2+1) \mathrm{D}$ model, which we consider next.

To obtain the defect formation energy and its dependence on the system size in two-dimensional space, we extended the one-dimensional model in two dimensions in a rotationally symmetric manner. We analytically solve the $(2+1) \mathrm{D}$ model, as shown in the Supplemental Material (SM) [9], and find that for FF boundary conditions the energy scales as $\sim 1 / \log (N)$ with system size. Furthermore, at DF boundary conditions, the force scales as $f \sim 1 / \sqrt{\log (N)}$, whereas the energy scales as $E \sim 1 / \log (N)$ with a constant offset, which is the formation energy of the defect in the thermodynamic limit. In Fig. 1(c), we show the numerical calculations of both the energy and force within this simple model. The data points are fitted with analytical predictions, and show very good agreement. The most striking result here is that both boundary conditions yield finite-size corrections of the form $1 / \log (N)$, on top of a constant offset. In the limit of infinite system size, FF and DF boundaries therefore yield different formation energies. This very simple model captures an essential feature of the formation energy of a lattice defect in a buckled two-dimensional crystal, which is its dependence on the boundary conditions. Furthermore, the same model also produces the finite-size scaling of the energy as found in our computer simulations on graphene, which we present next.

To further demonstrate this effect, we numerically study the formation energy of a single Stone-Wales (SW) defect, made of a pair of pentagon-heptagon rings obtained when four hexagons are transformed by a bond transposition of $90^{\circ}$, 
(a)

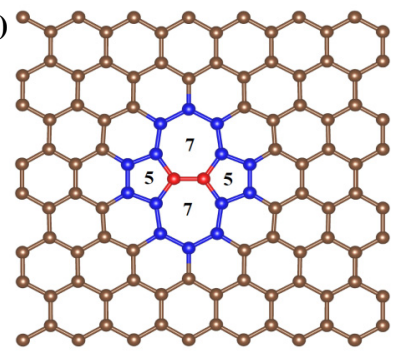

(c)

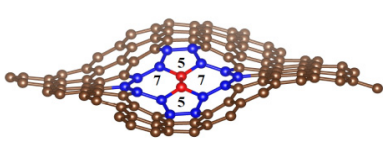

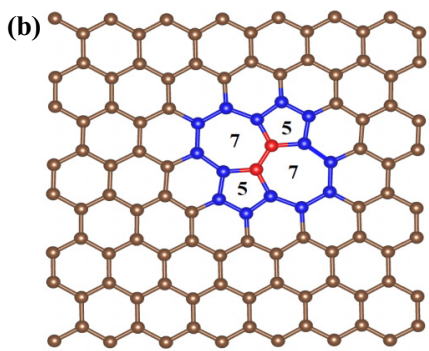

(d)

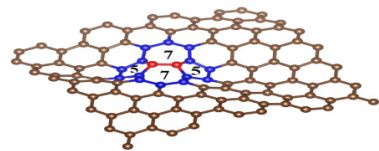

FIG. 2. Structure of the graphene sample with a single SW defect. The two different orientations of the defect are shown: (a) $0^{\circ}$ and (b) $60^{\circ}$. Two different buckling modes represent (c) sine-type buckling and (d) cosine-type buckling. The two configurations are shown from different viewing angles.

in a graphene sheet buckled in the out-of-plane direction, as shown in Fig. 2. We consider FF and DF boundary conditions, both of which are periodic as commonly used in simulations. Our results show that with DF boundaries the formation energy for the SW defects is always significantly higher than with FF boundaries, and such boundaries therefore strongly favor defect-free configurations of buckled graphene samples. Contrary to the natural intuition, the energy difference persists in the thermodynamic (infinite size) limit, as shown in Fig. 3, even though all individual atomic positions become indistinguishable between the two types of boundaries. In finite-size samples, this gap is more pronounced, as is especially the case for separated dislocations with FF versus DF boundary conditions (Fig. S3 in the SM) [9], where it can exceed $10 \mathrm{eV}$. Finite-size effects remain even in very large samples, since the finite-size corrections in the energy decrease inversely proportional to the logarithm of the system size. In contrast, if all atoms are confined to a purely two-dimensional plane, both FF and DF boundaries quickly converge to the same formation energy which is much higher than in the buckled samples. Finite-size corrections in this case decrease much faster, inversely proportional to the system size. Apparently, buckling introduces strong finite-size effects, with boundary effects that do not vanish in the thermodynamic limit. Our results therefore imply that both the formation energy of the lattice defects and its dependence on the size of the buckled graphene samples are not well defined without specifying the boundary conditions, counter to the conventional wisdom [5].

We simulated structures of a graphene membrane with an SW defect. Structural relaxation and energy computation are based on a recently developed semiempirical elastic potential for graphene [10]. Eight different geometries were used in our simulations. They differ in the orientation of the SW defect relative to the boundaries $\left(0^{\circ}\right.$ and $\left.60^{\circ}\right)$, the buckling modes (sine and cosine), and the types of boundaries (DF and FF). The two inequivalent initial bonds give rise to the two $\mathrm{SW}$ defects oriented by $60^{\circ}$ relative to each other [Figs. 2(a)
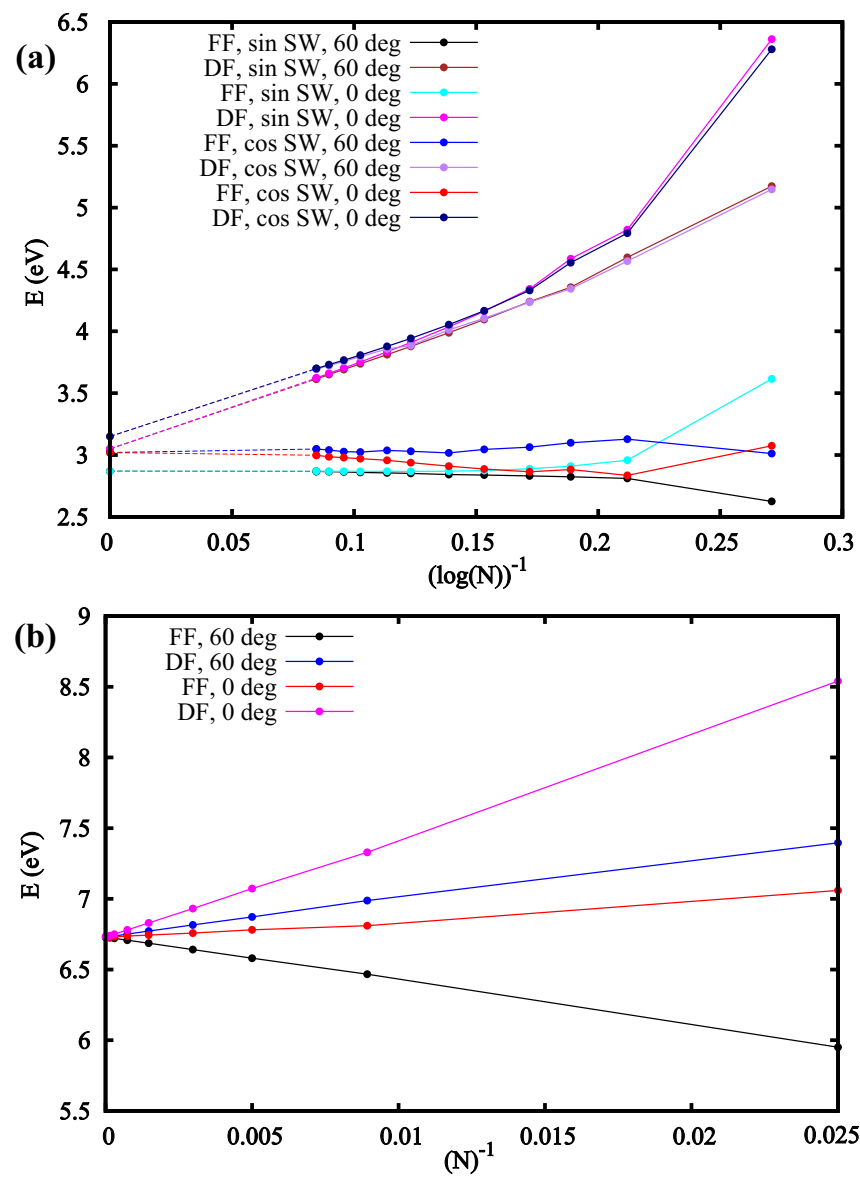

FIG. 3. Formation energy as a function of the graphene sample size with a single SW defect for both buckled (sine and cosine) and flat configurations, with different boundaries (DF and FF) and defect orientations $\left(0^{\circ}\right.$ and $\left.60^{\circ}\right)$. (a) In buckled graphene, the formation energy of the SW defect converges to four different values, determined by the boundary condition and buckling mode; different orientations do not influence the formation energy in the thermodynamic limit. Finite-size corrections scale as $1 / \log (N)$, with different prefactors for different boundaries, buckling modes, and defect orientations. (b) In flat graphene, the formation energy of the SW defect converges to the same value $(6.73 \mathrm{eV})$ irrespective of the orientation or the boundary condition, with finite-size corrections scaling as $1 / N$.

and 2(b)]. The system is then relaxed and to relieve the stress it buckles perpendicularly to the flat graphene plane with the two possible buckling configurations, sine and cosine [Figs. 2(c) and 2(d)], while the density of carbon atoms is kept fixed (DF) and relaxed (FF) (only FF shown in Fig. 2).

The calculated formation energies of a single SW defect in a buckled graphene sheet for different system sizes are shown in Fig. 3. Its scaling with the system size is given by

$$
E_{\mathrm{SW}}(N)=E_{0}+F(N),
$$

where $E_{0}$ is the energy contribution of the defect in an infinite (square) system, and $F(N)$ describes finite-size corrections, with lateral sample size $L$ and a number of carbon atoms $N \sim L^{2}$. For the computational methods, see the SM [9]. We first observe for the eight structures that extrapolation to the infinite system size produces four different values for the 
TABLE I. Formation energy $E_{0}$ of an SW defect in graphene in the thermodynamic limit, and form of the corresponding leading finite-size corrections for different orientations $\left(0^{\circ}\right.$ or $60^{\circ}$ with respect to the periodic directions; see Figs. 2(a) and 2(b)], different buckling [sine or cosine; see Figs. 2(c) and 2(d)], and different boundaries (FF or DF). Note that $E_{0}$ does not depend on defect orientation, but does depend on the buckling mode as well as on the type of boundary conditions. The leading finite-size correction in the formation energy scales as $1 / \log (N)$, with varying amplitude. The lowest formation energy $(2.87 \mathrm{eV})$ is for the configuration with $\mathrm{FF}$ boundaries and sine-type buckling, whereas the highest $(3.15 \mathrm{eV})$ is for the DF boundaries with cosine-type buckling. Most importantly, the formation energies in the thermodynamic limit for DF and FF boundaries differ by $0.18 \mathrm{eV}$ for sine-type buckling.

\begin{tabular}{lcc}
\hline \hline Defect and boundary type & $E_{0}(\mathrm{eV})$ & $F(N)$ \\
\hline $\mathrm{FF}, \sin \mathrm{SW}, 60^{\circ}$ & 2.87 & $1 / \sqrt{N^{\mathrm{a}}}$ \\
$\mathrm{DF}, \sin \mathrm{SW}, 60^{\circ}$ & 3.05 & $1 / \log (N)$ \\
$\mathrm{FF}, \sin \mathrm{SW}, 0^{\circ}$ & 2.87 & $1 / \sqrt{N}^{\mathrm{a}}$ \\
$\mathrm{DF}, \sin \mathrm{SW}, 0^{\circ}$ & 3.05 & $1 / \log (N)$ \\
$\mathrm{FF}, \cos \mathrm{SW}, 60^{\circ}$ & 3.02 & $1 / \sqrt{N^{\mathrm{a}}}$ \\
$\mathrm{DF}, \cos \mathrm{SW}, 60^{\circ}$ & 3.15 & $1 / \log (N)$ \\
$\mathrm{FF}, \cos \mathrm{SW}, 0^{\circ}$ & 3.02 & $1 / \sqrt{N} \mathrm{a}$ \\
DF, $\cos \mathrm{SW}, 0^{\circ}$ & 3.15 & $1 / \log (N)$ \\
\hline \hline
\end{tabular}

${ }^{\text {a In the case of FF boundaries, finite-size corrections for sample sizes }}$ studied here (up to 137616 atoms) are dominated by the scaling factor of $1 / \sqrt{N}$ [10], but a correction $\sim 1 / \log (N)$ with a small prefactor cannot be excluded.

formation energy $E_{0}$ of the defect; the dependence on the orientation of the defect vanishes, in agreement with the intuitive expectation based on the equivalence of the $s p^{2}$ carbon bonds. On the other hand, the defect energy depends on both the buckling configuration, and most notably, on the type of boundary of the sample. In particular, the DF boundaries, in which the density of the carbon atoms is fixed to the crystalline value, always give a higher formation energy of the defect than FF boundaries (see Table I). Therefore, boundary conditions play a crucial role in determining the formation energy of the defects.

This effect is especially pronounced when taking into account the finite size of the graphene samples. As shown in Fig. 3(a), there is a notable difference in the formation energy of the SW defects of up to $30 \%$ between the samples with DF and FF boundaries at the size of $N \sim 10^{4}$ atoms. More importantly, the finite-size correction to the defect energy, $F(N)$, scales as $1 / \log N$ for DF boundaries. Therefore, DF boundaries besides giving higher formation energy of the defects in the thermodynamic limit, also give rise to its slow decrease with the system size. On the other hand, as shown in Fig. 3(b), when the buckling is completely suppressed, the energy of the defect in the thermodynamic limit converges to a common value independently of the type of boundaries, with finite-size correction $F(N)=C / N$ in which the prefactor $C$ differs for both types of boundaries and the defect orientations. Notice also that in the flat graphene sheet, the DF boundaries give the largest energy for the defect formation in finite-size samples.
The effects of the boundaries are even more pronounced when considering the energy of a dislocation pair as a function of the distance (see SM [9]). The size of the energy difference between the FF and DF boundaries for a dislocation pair can be of the order of $10 \mathrm{eV}$ (Fig. S3). Moreover, the form of the potential between the dislocations depends heavily on the type of boundaries, implying a strong dependence of the melting temperature of graphene $[11,12]$ on the boundary conditions. For FF boundary conditions the energy of a dislocation pair as a function of separation in a buckled graphene membrane quickly becomes constant as predicted by Seung and Nelson in the inextensional limit [13]. The strain field around the core of a dislocation becomes short ranged when buckling is allowed and therefore the energy converges to a finite value. On the other hand, for DF boundary conditions the energy of a dislocation pair in the buckled crystal increases with separation and this behavior is consistent with the results obtained from a different elastic potential $[11,14]$. The increase in the energy in this case is lower than logarithmic, as predicted by Seung and Nelson. The strain field around the core of a dislocation does not become localized in the case of DF boundaries since a constant stretching force is applied at the boundaries in order to keep the atom density fixed and this could be the origin of the boundary effects. Furthermore, the force at the boundaries decreases with increasing system size, but at the same time the length of the boundary increases, and the combined effect on the defect's energy apparently is a constant offset as shown by our $(2+1) \mathrm{D}$ analytical model and numerical simulations on graphene.

Another qualitative way to understand our results, which at first glance seem surprising, is that a defect such as $\mathrm{SW}$, locally deforms the membrane thereby reducing the "footprint" in the 2D plane. With FF boundary conditions, the system can simply shrink to the reduced footprint, but with DF boundary conditions it cannot, resulting in significant stress. The latter raises the energy, even in the thermodynamic limit.

Our work demonstrates the crucial importance of boundaries for determining the formation energy of the lattice defects. Boundary effects may also be partly responsible for the large variation of the reported formation energies of defects in numerical simulations on the graphene lattice [5,15]. Simple models for an elastic string and a membrane embedded in a higher-dimensional space suggest their independence of the lattice geometry and the model, and in that sense they may represent a universal feature of the low-dimensional buckled crystals. Our findings may be relevant to graphene samples where SW defects [16-18] and grain boundaries have been observed [19-21]. Finally, our study opens up a route to investigate this boundary effect on the defects' energy in other two-dimensional crystalline materials, such as $\mathrm{Mo}_{2} \mathrm{C}$ [22], as well as in recently synthesized silicene $[23,24]$, germanene [25], and stanene [26].

We acknowledge the financial support by the FOMSHELL-CSER program (12CSER049). We are grateful to M. van Huis and J. Zaanen for critical reading of the manuscript, and M. Katsnelson for useful discussions. 
[1] J.-H. Chen, W. G. Cullen, C. Jang, M. S. Fuhrer, and E. D. Williams, Defect Scattering in Graphene, Phys. Rev. Lett. 102, 236805 (2009).

[2] A. Zandiatashbar, G.-H. Lee, S. J. An, S. Lee, N. Mathew, M. Terrones, T. Hayashi, C. R. Picu, J. Hone, and N. Koratkar, Effect of defects on the intrinsic strength and stiffness of graphene, Nat. Commun. 5, 3186 (2014).

[3] G. Lopez-Polin, C. Gomez-Navarro, V. Parente, F. Guinea, M. Katsnelson, F. Pérez-Murano, and J. Gómez-Herrero, Increasing the elastic modulus of graphene by controlled defect creation, Nat. Phys. 11, 26 (2014).

[4] R. Grantab, V. B. Shenoy, and R. S. Ruoff, Anomalous strength characteristics of tilt grain boundaries in graphene, Science 330 , 946 (2010)

[5] S. T. Skowron, I. V. Lebedeva, A. M. Popov, and E. Bichoutskaia, Energetics of atomic scale structure changes in graphene, Chem. Soc. Rev. 44, 3143 (2015).

[6] A. Fasolino, J. H. Los, and M. I. Katsnelson, Intrinsic ripples in graphene, Nat. Mater. 6, 858 (2007).

[7] F. Banhart, J. Kotakoski, and A. V. Krasheninnikov, Structural defects in graphene, ACS Nano 5, 26 (2011).

[8] T. Zhang, X. Li, and H. Gao, Defects controlled wrinkling and topological design in graphene, J. Mech. Phys. Solids 67, 2 (2014).

[9] See Supplemental Material at http://link.aps.org/supplemental/ 10.1103/PhysRevB.94.020102 for (1) detailed derivations of $(1+1) \mathrm{D}$ and $(2+1) \mathrm{D}$ analytical models; (2) computational details for numerical simulation of graphene; and (3) dislocation separation potential.

[10] S. K. Jain, G. T. Barkema, N. Mousseau, C.-M. Fang, and M. A. van Huis, Strong long-range relaxations of structural defects in graphene simulated using a new semiempirical potential, J. Phys. Chem. C 119, 9646 (2015).

[11] J. H. Los, K. V. Zakharchenko, M. I. Katsnelson, and A. Fasolino, Melting temperature of graphene, Phys. Rev. B 91, 045415 (2015).

[12] K. V. Zakharchenko, A. Fasolino, J. H. Los, and M. I. Katsnelson, Melting of graphene: From two to one dimension, J. Phys.: Condens. Matter 23, 202202 (2011).

[13] H. S. Seung and D. R. Nelson, Defects in flexible membranes with crystalline order, Phys. Rev. A 38, 1005 (1988).

[14] J. M. Carlsson, L. M. Ghiringhelli, and A. Fasolino, Theory and hierarchical calculations of the structure and energetics of [0001] tilt grain boundaries in graphene, Phys. Rev. B 84, 165423 (2011).

[15] E. Ertekin, D. C. Chrzan, and M. S. Daw, Topological description of the Stone-Wales defect formation energy in carbon nanotubes and graphene, Phys. Rev. B 79, 155421 (2009).

[16] A. Hashimoto, K. Suenaga, A. Gloter, K. Urita, and S. Iijima, Direct evidence for atomic defects in graphene layers, Nature (London) 430, 870 (2004).

[17] J. C. Meyer, C. Kisielowski, R. Erni, M. D. Rossell, M. F. Crommie, and A. Zettl, Direct imaging of lattice atoms and topological defects in graphene membranes, Nano Lett. 8, 3582 (2008).

[18] J. Kotakoski, A. V. Krasheninnikov, U. Kaiser, and J. C. Meyer, From Point Defects in Graphene to Two-Dimensional Amorphous Carbon, Phys. Rev. Lett. 106, 105505 (2011).

[19] P. Y. Huang, C. S. Ruiz-Vargas, A. M. van der Zande, W. S. Whitney, M. P. Levendorf, J. W. Kevek, S. Garg, J. S. Alden, C. J. Hustedt, Y. Zhu et al., Grains and grain boundaries in single-layer graphene atomic patchwork quilts, Nature (London) 469, 389 (2011).

[20] O. V. Yazyev and Y. P. Chen, Polycrystalline graphene and other two-dimensional materials, Nat. Nanotechnol. 9, 755 (2014).

[21] A. W. Tsen, L. Brown, R. W. Havener, and J. Park, Polycrystallinity and stacking in CVD graphene, Acc. Chem. Res. 46, 2286 (2013).

[22] C. Xu, L. Wang, Z. Liu, L. Chen, J. Guo, N. Kang, X.-L. Ma, H.-M. Cheng, and W. Ren, Large-area high-qality 2D ultrathin $\mathrm{Mo}_{2} \mathrm{C}$ superconducting crystals, Nat. Mater. 14, 1135 (2015).

[23] P. Vogt, P. De Padova, C. Quaresima, J. Avila, E. Frantzeskakis, M. C. Asensio, A. Resta, B. Ealet, and G. Le Lay, Silicene: Compelling Experimental Evidence for Graphenelike TwoDimensional Silicon, Phys. Rev. Lett. 108, 155501 (2012).

[24] B. Feng, Z. Ding, S. Meng, Y. Yao, X. He, P. Cheng, L. Chen, and $\mathrm{K} . \mathrm{Wu}$, Evidence of silicene in honeycomb structures of silicon on $\mathrm{Ag}(111)$, Nano Lett. 12, 3507 (2012).

[25] E. Bianco, S. Butler, S. Jiang, O. D. Restrepo, W. Windl, and J. E. Goldberger, Stability and exfoliation of germanane: A germanium graphane analogue, ACS Nano 7, 4414 (2013).

[26] F.-f. Zhu, W.-j. Chen, Y. Xu, C.-l. Gao, D.-d. Guan, C.-h. Liu, D. Qian, S.-C. Zhang, and J.-f. Jia, Epitaxial growth of twodimensional stanene, Nat. Mater. 14, 1020 (2015). 